\title{
Editorial
}

Von den vier Beiträgen der vorliegenden Ausgabe lässt sich einer dem Bereich der Personalwirtschaft zuordnen, zwei stammen aus dem Gebiet des Marketing und einer ist an der Nahtstelle zwischen Investition und Produktion angesiedelt.

\section{Mitarbeiterrekrutierung über das Internet}

Durch das Internet haben sich die Möglichkeiten der Mitarbeiterrekrutierung erheblich erweitert. Wie sie ausgestaltet ist und zu welchen Konsequenzen sie führt, ist in der Literatur bislang vornehmlich aus der Sicht des suchenden Unternehmens beschrieben und analysiert worden. Dagegen fehlt es an Untersuchungen aus der Perspektive der suchenden Arbeitnehmer. Dieses Defizit möchte Grund mit seinem Beitrag beheben, indem er eine Marktanalyse zur Mitarbeiterrekrutierung über das Internet vorstellt und sie mit einer empirischen Untersuchung über Determinanten und Konsequenzen für die Arbeitnehmer verknüpft. Konkret geht es um die Frage, welche Personen neue Arbeitsplätze über das Internet suchen und finden und welche Auswirkungen sich dadurch für die Arbeitnehmer ergeben. Die Marktbeschreibung für die Bundesrepublik Deutschland fördert zutage, dass hierzulande etwa 10 Prozent der Personen mit Internetzugang einen Arbeitsplatz über dieses Medium suchen; in den USA sind es dem gegenüber bereits 17 Prozent. Als Formen des Kontakts zwischen Arbeitgeber und Arbeitnehmer werden Darstellungen auf den Homepages der Unternehmungen, Jobbörsen und Bewerberdatenbanken untersucht. Eine schöne Marktübersicht zeigt, wie unter diesen Formen die Anzahl der angebotenen Jobs, die Seitenaufrufe pro Monat, die Kosten pro Anzeige, die Anzahl der Bewerber und die Zugriffskosten verteilt sind. Der Autor untersucht des Weiteren die Vor- und Nachteile der Mitarbeiterrekrutierung über das Internet im Vergleich zu Inseraten in der Zeitung. Die wesentlichsten Vorteile liegen in der Zeitersparnis, den geringeren Kosten und der Tatsache, dass sich über das Internet mehr potentielle Bewerber pro Vakanz melden als über Inserate. Nachteile liegen darin, dass Personen ohne Internetzugang durch Stellenangebote nicht erreicht werden und bei der Rekrutierung über das Internet der Anteil ungeeigneter Bewerber höher ausfällt als bei der Ausschreibung über Inserate. Die empirische Studie fußt auf der Datengrundlage des sozio-ökonomischen Panels und wertet die Ergebnisse der letzten Befragung vom Jahre 2003 aus. Demnach suchen eher jüngere Personen mit besserer Schulausbildung nach einem Arbeitsplatz über das Internet. Diese Arbeitsplätze sind eher in größeren Unternehmen angesiedelt und durch eine relativ höhere Wochenarbeitszeit charakterisiert. Personen, die ihren Arbeitsplatz über das Internet suchen, verdienen mitunter mehr. Im Hinblick auf die Arbeitszufriedenheit haben sich im Vergleich zu anderen Wegen der Stellenfindung keine Unterschiede in Abhängigkeit verschiedener individueller und arbeitsplatzspezifischer Charakteristika gezeigt.

\section{Determinanten der Informationsfunktion des Preises}

$\mathrm{Zu}$ Beginn ihrer empirischen Analyse weist Völkner darauf hin, dass es in der Literatur zwar bereits eine intensive Forschung zu der Frage gibt, unter welchen Bedingungen Kon- 
sumenten dazu neigen, von einem höheren Preis des Produktes auf eine höhere Produktqualität zu schließen, diese Untersuchungen jedoch insofern unbefriedigend bleiben, als sie die relative Bedeutung der Einflussgrößen nicht klar herausarbeiten und die Befunde oft widersprüchlich sind. So beschreitet die Autorin in ihrem Beitrag erstmals methodisch den Weg, eine simultane Untersuchung einer Vielzahl von Determinanten vorzunehmen, wodurch Aussagen zur Signifikanz und relativen Wirkungsstärke der Einflussgrößen gewonnen werden können. Die umfassende Studie bezieht insgesamt 17 potentielle Determinanten der Informationsfunktion des Preises in die Überlegungen ein, welche sich aus Erkenntnissen bisheriger Forschungen als nahe liegend anbieten. Dabei wird zwischen personenbezogenen, produktkategoriebezogenen und situativen Faktoren unterschieden. Im Detail sollen sie die Einflüsse erfassen, welche das Qualitätsinteresse und der Kenntnisstand des Kunden sowie der Zeitdruck beim Einkauf auf eine preisorientierte Qualitätsbeurteilung des Produktes ausüben. In der empirischen Studie wurden 314 haushaltsführende Konsumenten danach befragt, aus welchen Gründen sie beim Kauf des kurzlebigen Konsumgutes Konfitüre geneigt sind, vom Preis des Produktes auf dessen Qualität zu schließen. Als signifikante Determinanten haben sich beispielsweise das Sparsamkeitsstreben, das Exklusivitätsstreben, das Prestigebedürfnis, das Qualitätsinteresse und die Komplexität der Einkaufsaufgabe sowie die von den Konsumenten wahrgenommenen Qualitätsunterschiede in der jeweiligen Produktkategorie herausgestellt. Der Kenntnisstand der Konsumenten in der jeweiligen Produktkategorie hatte einen signifikant negativen Einfluss. Die situativen Faktoren wurden nicht als bedeutungsvoll erkannt. Die Ergebnisse wurden mithilfe einer multivariaten Regressionsanalyse hergeleitet. Um anschließend Erkenntnisse darüber zu gewinnen, inwieweit die Heterogenität innerhalb der Gruppe der Konsumenten zur unterschiedlichen Signifikanz von Determinanten führt, hat die Autorin zudem eine Latent-Class-Regressionsanalyse durchgeführt, mit deren Hilfe diese Zusammenhänge studiert werden. Dabei offenbaren sich zwei Konsumentensegmente mit unterschiedlicher Signifikanz der Determinanten. Für die praktische Preisbestimmung in Unternehmungen sind die Charakteristika interessant, durch welche die Konsumentensegmente gekennzeichnet sind. Das eine Segment wird eher von jungen Leuten im Alter von 14 bis 34 Jahren gebildet, die eher den Ergebnissen der Stiftung Warentest vertrauen. Das andere Segment wird eher von Personen im Alter von 35 bis 70 Jahren gebildet, die eine signifikant höhere Tendenz zur preisorientierten Qualitätsbeurteilung und eine höhere Zahlungsbereitschaft aufweisen. Das zweite Segment bestätigt eher die Ergebnisse der multivariaten Regressionsanalyse; das erste Segment zeigt dagegen ein etwas anderes Bild der Signifikanz der Determinanten. Bedeutung erhalten diese Erkenntnisse für die zielgruppenspezifische Gestaltung der Informationsfunktion des Preises.

\section{Planung effektiver und effizienter Werbemixes}

Die analytische Arbeit von Steffenhagen leistet einen Beitrag zur Frage der optimalen Gestaltung des Werbemixes. Damit wird eine theoretische Erörterung wieder aufgegriffen, die vor über 20 Jahren vornehmlich im Hinblick auf die Ziele, durch den optimalen Werbemix den Gewinn bzw. die Anzahl der Werbekontakte zu maximieren, schon einmal intensiv geführt worden ist. Allerdings - so die Argumentation des Autors - greift das 
Gewinnziel zu weit und das Kontakteziel zu kurz, weil sich in der Praxis die Aufteilung des Gesamtwerbeetats auf die Werbeinstrumente an den vom Unternehmen verfolgten psychographischen Werbezielen orientieren muss und dazu vor allem Erkenntnisse der Marketing-Mix-Wirkungsanalyse und -prognose erforderlich sind. Die Ausrichtung der optimalen Planung des Werbemixes an den so genannten ,außerökonomischen Werbezielen“ der Effektivität und Effizienz, mit deren Hilfe die Güte der Erreichung angestrebter Ziele und ihrer wirtschaftlichen Erfüllung beurteilt werden sollen, impliziert jedoch keineswegs den Verzicht auf eine ökonomische Theorie des Werbemixes. Zu dieser Theorie entwickelt der Autor Bausteine, die auf dem Ansatz einer multiplikativen Werbewirkungsfunktion - analog zu einer Produktionsfunktion vom Typ Cobb/Douglas - aufbauen. Als psychographische Werbeziele kommen die Erinnerung, die Aufmerksamkeit und die Verhaltensbereitschaft in Bezug auf ein beworbenes Produkt in Frage. Sie weisen Ähnlichkeiten zu Aspekten auf, wie sie auch aus der AIDA-Formel her bekannt sind. Der spezielle Typ der multiplikativen Werbewirkungsfunktion erlaubt es, nach Logarithmierung der Funktion die Wirkungszusammenhänge auf der Grundlage einer linearen Regression zu untersuchen, wobei Messwerte aus dem Werbetracking der Praxis benutzt werden können. Als spezielle Planungssituationen einer statischen Werbemixplanung werden die Fälle unterschieden, ob der Werbeetat offen oder begrenzt ist bzw. ob die Werbeziele in ihrem Niveau offen oder vorgegeben sind. Die Erweiterung der Analyse auf eine dynamische Werbemixplanung geht der Frage nach, wie die Werbeimpulse dosiert sein sollen. Dabei werden Fälle der Wirkungsverzögerung, der Wirkungsübertragung sowie des zeitlichen Wirkungsverbundes unterschieden. Speziell für den Fall der Wirkungsübertragung wird ein theoretischer Ansatz vorgestellt und durch ein numerisches Beispiel belegt. Hier kann allgemein ein Optimalitätstheorem zur mehrstufigen dynamischen Werbemixplanung hergeleitet werden. Konkret empfiehlt es sich, nach eingelegten Werbepausen eine speziell gewünschte psychographische Werbewirkung dadurch zu erzielen, dass der Werbeetat über die Zeit progressiv ansteigt.

\section{Umweltzertifikate und Investitionen}

In seinem analytischen Beitrag untersucht Klingelhöfer, inwieweit Umweltzertifikate einen Anreiz für Investitionen in den Umweltschutz bewirken. Um dies beurteilen zu können, müssen die produktionswirtschaftlichen Zusammenhänge und deren monetäre Konsequenzen in die Zahlungsströme des Investitionskalküls einbezogen werden. Dann lassen sich die Auswirkungen von Änderungen in den Zertifikatspreisen und -mengen auf die Vorteilhaftigkeit von Umweltinvestitionen mit Hilfe der Sensitivitätsanalyse analysieren. Zu diesem Zweck wird ein zweistufiger Bewertungsansatz verwendet, um den Grenzpreis zu bestimmen, für den sich die Durchführung der Investition gerade noch lohnt. Dualitätstheoretische Betrachtungen erlauben die Einbeziehung von Zertifikaten in Partialmodelle wie beispielsweise bei der Anwendung der Kapitalwertmethode. Zunächst werden die Konsequenzen von Umweltzertifikaten für die Programmplanung studiert. Ein zweistufiger Bewertungsansatz auf der Grundlage der linearen Programmierung gestattet es dann, Umweltschutzinvestitionen unter dem Aspekt des Zertifikathandels zu beurteilen. In der ersten Stufe werden in einem Basisprogramm die Investitionsauswirkungen 
ohne die Durchführung der entsprechenden Umweltschutzinvestition betrachtet. Das Ergebnis ist plausibel, dass von Unternehmungen dann Umweltzertifikate verkauft werden, wenn der Zertifikatspreis höher ist als die Kosten, die mit einer Investition in die Emissionsreduktion des Unternehmens verbunden sind. Auf der zweiten Stufe wird dann durch ein Bewertungsprogramm abgeschätzt, inwieweit sich eine Umweltschutzinvestition lohnt. Da mit der Investition bzw. durch deren Unterlassung auch immer eine Beeinflussung des Zertifikatspreises einhergeht, kann es - wie die analytischen Betrachtungen zeigen sowohl dazu kommen, dass Zertifikate eine Umweltinvestition günstiger erscheinen lassen, oder aber auch gleichwohl kontraproduktive Wirkungen erzeugen können, d.h. durch die Einführung und Verschärfung einer Zertifikatslösung die Unterlassung von Umweltinvestitionen vorteilhafter wird.

\section{Das Juni-Heft}

Im nächsten Heft der ZfB gelangen wieder vier Beiträge zum Abdruck. Sie beziehen sich auf die Gebiete Wirtschaftsinformatik, Unternehmensführung, Wirtschaftsprüfung sowie Bankwirtschaft.

Heinrich und Klier stellen einen Optimierungsansatz für ein fortlaufendes Datenqualitätsmanagement und dessen praktische Anwendung bei Kundenkampagnen eines Mobilfunkanbieters vor. Der Beitrag gibt einen Überblick über Ansätze zur ökonomischen Analyse des Datenqualitätsmanagements. Danach wird ein Optimierungsmodell entwickelt, das als Grundlage für ein effizientes, fortlaufendes Datenqualitätsmanagement dient. Hieraus lassen sich dann konkrete Handlungsempfehlungen für die Praxis ableiten.

Der Einfluss der Unternehmensgröße sowie der Eigentums- und Führungsstruktur auf die Steuerung mittelständischer Unternehmen wird in der Arbeit von Schachner, Speckbacher und Wentges untersucht. Insbesondere zeigt sich, dass Eigentümerfamilien und deren Mitwirkung in der Geschäftsführung einen entscheidenden Einfluss auf die Art der Unternehmenssteuerung haben.

Die Qualität der Nachhaltigkeitsberichterstattung wird von Quick und Knocinski anhand empirischer Befunde zur Berichterstattungspraxis von HDAX-Unternehmen untersucht. Die Nachhaltigkeit bezieht sich dabei auf den Aspekt, inwieweit ökonomische, ökologische und soziale Einflussgrößen in das unternehmerische Handeln einbezogen werden. Die Güte der Nachhaltigkeitsberichterstattung wird mithilfe eines Scoringmodells bewertet.

Inwieweit die Ausgestaltung des bankinternen Ratingverfahrens als Ansatzpunkt zur Risikooptimierung zu dienen vermag, wird in dem Beitrag von Hofmann untersucht. Auf der Grundlage empirischer Ergebnisse wird eine modelltheoretische Analyse der Kreditbetreuersteuerung durchgeführt. Sie soll es ermöglichen, Anreizkonflikte zwischen Bankmanagement und Kreditmitarbeitern zu lösen. Ziel ist es, Fehleinschätzungen durch Bankmitarbeiter bei der Kreditvergabe zu vermeiden und dadurch das Ausfallrisiko zu optimieren.

Günter Fandel

Editor-in-Chief 